\title{
Pseudo-CSA Restraints for NMR Refinement of Nucleic Acid Structure
}

\author{
Alexander Grishaev, Jinfa Ying, and Ad Bax* \\ Laboratory of Chemical Physics, National Institute of Diabetes and Digestive and Kidney Diseases, \\ National Institutes of Health, Bethesda, Maryland 20892
}

Received May 11, 2006; E-mail: bax@nih.gov

Residual dipolar couplings (RDCs) provide global orientational restraints that have proven to be particularly useful in NMR structural studies of oligonucleotides, which frequently lack tertiary contacts. ${ }^{1-7}$ One-bond ${ }^{13} \mathrm{C}-{ }^{1} \mathrm{H}$ RDCs can be measured when the macromolecule of interest is weakly aligned relative to the magnetic field, ${ }^{8,9}$ most commonly accomplished by means of a dilute, aqueous lyotropic liquid crystalline medium consisting of bicelles ${ }^{10}$ or filamentous bacteriophage, ${ }^{1}$ or by use of anisotropically compressed acrylamide gels. 5,11,12 The values of the RDCs, including their sign, are extracted from the difference in the apparent ${ }^{1} J_{\mathrm{CH}}$ splittings between aligned and isotropic sample conditions. For oligonucleotides, the least resonance overlap in the $2 \mathrm{D}{ }^{1} \mathrm{H}-{ }^{13} \mathrm{C}$ spectrum, from which these couplings are extracted, is commonly found for the base correlations, where $\mathrm{C}_{2}, \mathrm{C}_{5}, \mathrm{C}_{6}$, and $\mathrm{C}_{8}$ each fall in their own characteristic regions. However, although the downfield ${ }^{13} \mathrm{C}-\left\{{ }^{1} \mathrm{H}\right\}$ doublet component is enhanced in resolution as a result of interference between the ${ }^{13} \mathrm{C}$ chemical shift anisotropy (CSA) and ${ }^{13} \mathrm{C}-{ }^{1} \mathrm{H}$ dipolar relaxation mechanisms, ${ }^{13}$ often referred to as the TROSY effect, ${ }^{14}$ the upfield doublet component has much larger line width. The concomitant increase in resonance overlap and weaker intensity of the upfield ${ }^{13} \mathrm{C}-\left\{{ }^{1} \mathrm{H}\right\}$ doublet component makes accurate measurement of its position more difficult, particularly in larger oligonucleotides, adversely affecting measurement of the corresponding RDC.

For nuclei with well characterized, uniform CSA tensors, such as backbone ${ }^{13} \mathrm{C}^{\prime}$ in proteins and ${ }^{31} \mathrm{P}$ in nucleic acids, experimentally determined chemical shift changes, $\Delta \delta$, between aligned and isotropic samples have proven to be useful orientational restraints in structure calculation, fully analogous to RDCs. ${ }^{15-17}$ The value of $\Delta \delta$ is given by

$$
\Delta \delta=\delta_{\text {aniso }}-\delta_{\text {iso }}=\sum_{i=X, Y, Z} \sum_{j=X, Y, Z} A_{j j} \cos ^{2}\left(\theta_{i j}\right) \delta_{i i}
$$

where $A_{j j}$ are the principal components of the molecular alignment tensor, $\delta_{i i}$ are the principal components of the CSA tensor, and $\theta_{i j}$ is the angle between principal axis $j$ of the alignment tensor and principal axis $i$ of the CSA tensor; $\Delta \delta$ is measured from the decoupled HSQC spectrum at a resolution and sensitivity that is intermediate between that of the narrow downfield and the broad upfield ${ }^{13} \mathrm{C}-\left\{{ }^{1} \mathrm{H}\right\}$ doublet component in a ${ }^{1} \mathrm{H}$-coupled HSQC spectrum. Thus, although intrinsically useful, ${ }^{18}$ accurate measurement of both RDCs and $\Delta \delta$ becomes difficult for larger oligonucleotides.

In contrast, the position of the narrow, downfield ${ }^{13} \mathrm{C}-\left\{{ }^{1} \mathrm{H}\right\}$ TROSY component can be determined accurately even for larger, slowly tumbling oligonucleotides. Its change in resonance position between isotropic and aligned conditions equals $\Delta \delta^{\prime}=\Delta \delta+\mathrm{RDC} /$ 2 . We here demonstrate that such values can be readily measured in a slowly tumbling oligonucleotide, and that their incorporation as restraints results in considerable improvement in structural

10010 - J. AM. CHEM. SOC. 2006, 128, 10010-10011

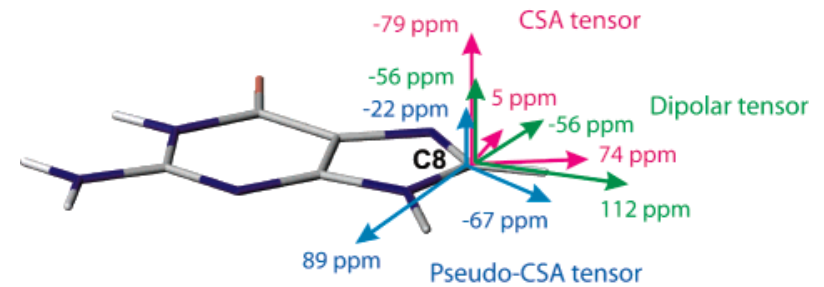

Figure 1. Schematic representation of the orientation and eigenvalues of the CSA tensor, $\mathrm{C}-\mathrm{H}$ dipolar interaction tensor, and TROSY pseudo-CSA tensor for $\mathrm{G}-\mathrm{C} 8$ at $800 \mathrm{MHz}{ }^{1} \mathrm{H}$ frequency.

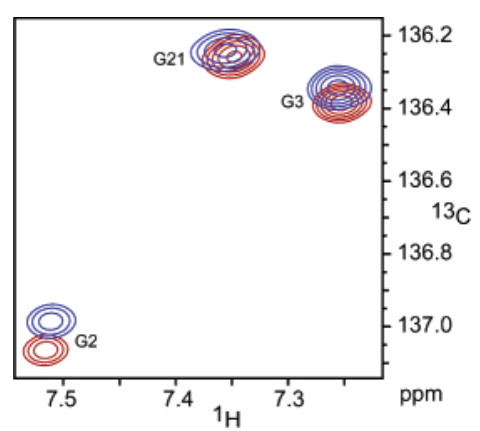

Figure 2. Superposition of a small region of the ${ }^{1} \mathrm{H}-{ }^{13} \mathrm{C}$ TROSY spectrum of helix-35, recorded under isotropic (red) and Pf1-aligned (blue) conditions, at $800 \mathrm{MHz}{ }^{1} \mathrm{H}$ frequency, $5^{\circ} \mathrm{C}$.

quality. Measurement of $\Delta \delta^{\prime}$ has previously been demonstrated for ${ }^{15} \mathrm{~N}-\left\{{ }^{1} \mathrm{H}\right\}$ in protein backbone amides. ${ }^{19}$ However, in that case, collinearity between the ${ }^{15} \mathrm{~N}$ CSA and the ${ }^{15} \mathrm{~N}-{ }^{1} \mathrm{H}$ dipolar tensors results in strong attenuation of the magnitude of $\Delta \delta^{\prime}$ relative to $\Delta \delta$ and makes $\Delta \delta^{\prime}$ quite sensitive to small site-to-site variations in CSA. Our recent experimental study ${ }^{20}$ of base ${ }^{13} \mathrm{C}$ CSA orientation and magnitude confirms that within helical secondary structure CSA tensors are quite uniform, highly rhombic, and have their most shielded component orthogonal to the plane of the base. ${ }^{21}$ Cancellation of dipolar and CSA effects therefore presents much less of a problem in nucleic acids than in proteins. In the frame of the base, the tensor describing the local field resulting from the sum of the dipolar and CSA interactions can be considered a "pseudo-CSA" tensor, obtained by adding an axially symmetric tensor describing the ${ }^{13} \mathrm{C}-{ }^{1} \mathrm{H}$ dipolar interaction to the regular CSA tensor. The magnitude and orientation of these pseudo-CSA tensors depend on the strength of the magnetic field (Figure 1).

Technically, the measurement of $\Delta \delta^{\prime}$ is quite straightforward, although particular care must be taken to ensure that the aligned and isotropic samples are as similar as possible in $\mathrm{pH}$, ionic strength, etc., as chemical shift changes resulting from such external factors could impact the measurement of $\Delta \delta^{\prime}$.

Figure 2 demonstrates the measurement of $\Delta \delta^{\prime}$ for a 24-nt stemloop RNA sequence, mimicking nucleotides 737-760 of E. coli 23S ribosomal RNA and modified to contain $\psi 746$. Experiments 

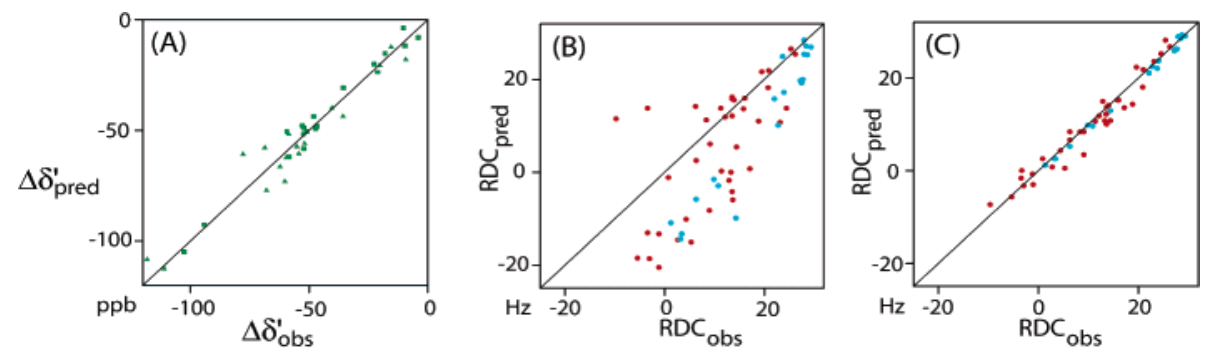

Figure 3. Agreement between experimental data and calculated structure of the A-form helical region of helix-35. (A) SVD fit of the observed TROSY $\Delta \delta^{\prime}$ shifts to the helix-35 coordinates (PDB code $2 \mathrm{GBH}$ ), using CSA parameters of ref 20 . Data measured at $25^{\circ} \mathrm{C}$ are shown as squares (rms $=3.7 \mathrm{ppb}$ ) and those measured at $5{ }^{\circ} \mathrm{C}$ as triangles $(\mathrm{rms}=4.3 \mathrm{ppb}$ ). (B,C) Cross-validation correlation plots, illustrating the agreement between observed and predicted one-bond ${ }^{13} \mathrm{C}-{ }^{1} \mathrm{H}$ RDCs with (C) and without (B) inclusion of $\Delta \delta^{\prime}$ shifts as restraints during structure calculation. Red and blue circles correspond to ribose and base RDCs, respectively. Root-mean-square differences (rmsds) between observed and predicted ${ }^{1} \mathrm{D}_{\mathrm{CH}}$ values decrease from $13.0 \pm 8.3 \mathrm{~Hz}$ when TROSY shifts are not fitted to $2.8 \pm 0.3 \mathrm{~Hz}$ when they are included in the structure calculation. Comparable relative improvements are observed in the absence of the empirical database: rmsds decrease from $18.1 \pm 6.2 \mathrm{~Hz}$ when TROSY shifts are not fitted to $4.3 \pm 0.9 \mathrm{~Hz}$ when included.

were carried out at $800 \mathrm{MHz}{ }^{1} \mathrm{H}$ frequency and $5{ }^{\circ} \mathrm{C}$, where ${ }^{13} \mathrm{C}$ relaxation rates indicate a rotational correlation time of $9.5 \pm 0.5$ ns. Outside the region shown in Figure 2, several correlations from the loop segment exhibit considerable $(>0.01 \mathrm{ppm}){ }^{1} \mathrm{H}$ chemical differences between aligned and isotropic samples, too large to be attributable to ${ }^{1} \mathrm{H} \mathrm{CSA}$. The loop is subject to dynamic averaging of multiple conformations, whose precise occupancies depend strongly on sample conditions. Even though both aligned and isotropic samples were extensively dialyzed against identical buffers, ${ }^{1} \mathrm{H}$ chemical shift changes $>0.01 \mathrm{ppm}$ remain, reflecting the effect of the Pf1 on the equilibrium of conformers and preventing reliable measurement of $\Delta \delta^{\prime}$ for these dynamically disordered residues. In contrast, with the exception of a terminal base pair adjacent to the loop, none of the helical nucleotides show ${ }^{1} \mathrm{H}$ chemical shift changes $>9 \mathrm{ppb}$, and their ${ }^{13} \mathrm{C} \Delta \delta^{\prime}$ values can be extracted reliably. These values closely agree with predictions made on the basis of the structure of the helical stem, previously solved by using a large number of RDCs measured at $25^{\circ} \mathrm{C}$ (Figure $3 \mathrm{~A})$. As a further test of the utility of using pseudo-CSAs in structure refinement, we recalculated the A-form helical region of helix-35, retaining only loose NOE and dihedral restraints as well as ${ }^{1} \mathrm{D}_{\mathrm{Cl}^{\prime}-\mathrm{H} 1^{\prime}} \mathrm{RDCs}$, with and without $\Delta \delta^{\prime}$ restraints. In both cases, database-derived base-base positioning potentials ${ }^{22}$ were employed since they were observed to improve cross-validation statistics. Comparison of Figure 3B and $\mathrm{C}$ shows a considerable improvement in prediction of both base and ribose RDCs upon incorporation of $\Delta \delta^{\prime}$ restraints. In the absence of the empirical base-base potential, even larger gains in cross-validation are observed (data not shown).

Measurement of $\Delta \delta^{\prime}$ and their incorporation as structural restraints is expected to be most useful for studies of larger structures that contain multiple helical elements, where RDCs can be difficult to measure but $\Delta \delta^{\prime}$ remains readily accessible. Importantly, besides improving local structure, these $\Delta \delta^{\prime}$ restraints tightly define the relative orientation of helical segments which often represents the major challenge by conventional NMR. Care must be taken when interpreting $\Delta \delta^{\prime}$ outside of helical regions, as minute changes in sample conditions associated with alignment can impact populations of any dynamic conformational equilibrium, often found in loop regions of such structures, thereby affecting chemical shifts. Moreover, the ${ }^{13} \mathrm{C} \mathrm{CSA}$ tensor itself depends on its local environment, ${ }^{20,21}$ and values applicable for bases outside the canonical A-form helical geometry are currently not accurately known.
Acknowledgment. We thank Ed Nikonowicz (Rice U.) for the helix-35 RNA sample, and Dennis Torchia (NIDCR, NIH) for helpful discussions. This work was supported by the Intramural Research Program of the NIDDK, NIH, and by the Intramural Antiviral Target Program of the Office of the Director, NIH.

Supporting Information Available: Two tables with ${ }^{1} \mathrm{H}$ and ${ }^{13} \mathrm{C}$ $\Delta \delta^{\prime}$ values measured at 25 and $5{ }^{\circ} \mathrm{C}$. Code for incorporating $\Delta \delta$ restraints into the structure calculation is available as a part of Xplor$\mathrm{NIH}$ distribution. This material is available free of charge via the Internet at http://pubs.acs.org.

\section{References}

(1) Mollova, E. T.; Hansen, M. R.; Pardi, A. J. Am. Chem. Soc. 2000, 122, $11561-11562$

(2) Bayer, P.; Varani, L.; Varani, G. J. Biomol. NMR 1999, 14, 149-155.

(3) Tjandra, N.; Tate, S.-i.; Ono, A.; Kainosho, M.; Bax, A. J. Am. Chem. Soc. 2000, 122, 6190-6200.

(4) Stefl, R.; Wu, H. H.; Ravindranathan, S.; Sklenar, V.; Feigon, J. Proc. Natl. Acad. Sci. U.S.A. 2004, 101, 1177-1182.

(5) Lukavsky, P. J.; Kim, I.; Otto, G. A.; Puglisi, J. D. Nat. Struct. Biol. 2003, 10, 1033-1038.

(6) D’Souza, V.; Dey, A.; Habib, D.; Summers, M. F. J. Mol. Biol. 2004, 337, 427-442.

(7) Al-Hashimi, H. M.; Pitt, S. W.; Majumdar, A.; Xu, W. J.; Patel, D. J. J Mol. Biol. 2003, 329, 867-873.

(8) Tolman, J. R.; Flanagan, J. M.; Kennedy, M. A.; Prestegard, J. H. Proc. Natl. Acad. Sci. U.S.A. 1995, 92, 9279-9283.

(9) Kung, H. C.; Wang, K. Y.; Goljer, I.; Bolton, P. H. J. Magn. Reson., Ser $B$ 1995, 109, 323-325.

(10) Tjandra, N.; Bax, A. Science 1997, 278, 1111-1114.

(11) Tycko, R.; Blanco, F. J.; Ishii, Y. J. Am. Chem. Soc. 2000, 122, 93409341.

(12) Sass, H.-J.; Musco, G.; Stahl, S. J.; Wingfield, P. T.; Grzesiek, S. J. Biomol. NMR 2000, 18, 303-309.

(13) Brutscher, B.; Boisbouvier, J.; Pardi, A.; Marion, D.; Simorre, J. P. J. Am. Chem. Soc. 1998, 120, 11845-11851.

(14) Pervushin, K.; Riek, R.; Wider, G.; Wuthrich, K. Proc. Natl. Acad. Sci. U.S.A. 1997, 94, 12366-12371.

(15) Lipsitz, R. S.; Tjandra, N. J. Am. Chem. Soc. 2001, 123, 11065-11066.

(16) Choy, W. Y.; Tollinger, M.; Mueller, G. A.; Kay, L. E. J. Biomol. NMR 2001, 21, 31-40.

(17) Wu, Z. R.; Tjandra, N.; Bax, A. J. Am. Chem. Soc. 2001, 123, 36173618 .

(18) Hansen, A. L.; Al-Hashimi, H. M. J. Magn. Reson. 2006, 179, 299-307.

(19) Tate, S.; Shimahara, H.; Utsunomiya-Tate, N. J. Magn. Reson. 2004, 171, 284-292.

(20) Ying, J.; Grishaev, A.; Bryce, D. L.; Bax, A. J. Am. Chem. Soc., in press.

(21) Stueber, D.; Grant, D. M. J. Am. Chem. Soc. 2002, 124, 10539-10551.

(22) Clore, G. M.; Kuszewski, J. J. Am. Chem. Soc. 2003, 125, 1518-1525. JA0633058 